\title{
Accuracy Limits of Distance Estimation in Visible Light Systems with RGB LEDs
}

\author{
Ilker Demirel \\ Department of Electrical \\ and Electronics Engineering \\ Bilkent University \\ Ankara, 06800, Turkey \\ Email: ilker.demirel@ug.bilkent.edu.tr
}

\author{
Sinan Gezici \\ Department of Electrical \\ and Electronics Engineering \\ Bilkent University \\ Ankara, 06800, Turkey \\ Email: gezici@ee.bilkent.edu.tr
}

\begin{abstract}
The distance estimation problem is investigated for visible light positioning (VLP) systems with red-green-blue (RGB) light emitting diodes (LEDs). The accuracy limits on distance estimation are calculated in terms of the Cramér-Rao lower bounds (CRLBs) for three different scenarios. Scenario 1 and Scenario 2 correspond to synchronous and asynchronous systems, respectively, with known channel attenuation formulas at the receiver. In Scenario 3, a synchronous systems is considered but channel attenuation formulas are not known at the receiver. The derived CRLB expressions reveal the relations among the distance estimation accuracies in the considered scenarios and provide intuitive explanations for the benefits of using RGB LEDs.
\end{abstract}

\section{INTRODUCTION}

Visible light positioning (VLP) systems have attracted significant attention in recent studies due to their lowcost and high-accuracy nature [1]. In addition, they incur almost no additional deployment cost as they are already employed for illumination. VLP systems with high localization accuracy can facilitate various applications such as robot tracking, patient monitoring, and warehouse management [2].

Among various theoretical and experimental studies related to VLP systems, a group of them focuses on determination of accuracy limits related to position estimation [3]-[8]. Accuracy limits provide theoretical performance bounds for a large class of estimators (such as unbiased estimators) and they can present guidelines for system design under certain accuracy requirements. In [3], the Cramér-Rao lower bound (CRLB) is obtained for distance estimation based on the time-ofarrival (TOA) parameter in a synchronous VLP system and the dependence of the CRLB on various system parameters is investigated. In [4], the CRLB is derived for distance estimation in an asynchronous VLP system, where the distance related information in the received signal strength (RSS) parameter is utilized. The work in [5] focuses on the distance estimation problem for both synchronous and asynchronous VLP systems, and considers the cases of known and unknown channel attenuation formulas at the visible light communication
(VLC) receiver. It is shown that the distance related information contained in the TOA parameter (which can be utilized in the presence of synchronization) increases with the effective bandwidth of the transmitted optical waveform. Therefore, synchronous VLP systems can provide performance improvements over asynchronous ones only for sufficiently high effective bandwidths. In addition to distance estimation, there also exist studies on localization in VLP system. For instance, [8] provides the CRLBs for three dimensional position estimation in synchronous and asynchronous VLP systems, and presents comparisons in various conditions.

Although the theoretical limits on distance/position estimation are investigated for VLP systems with white LEDs in [3]-[8], they are not available for VLP systems with red-green-blue (RGB) LEDs in the literature. Since RGB LEDs can provide additional benefits for visible light systems [9], [10], the analysis of theoretical limits is crucial for VLP systems with RGB LEDs, as well. The aim of this paper is to provide an initial step towards this direction by considering accuracy of distance estimation in VLP systems with RGB LEDs. In particular, the CRLBs on distance estimation are derived for the first time in the literature for both synchronous and asynchronous VLP systems with RGB LEDs. In addition, in the synchronous scenario, the cases of known and unknown channel attenuation formulas at the VLC receiver are considered. The provided CRLB expressions generalize the ones in the literature [5] as there exist three parallel channels in RGB based VLP systems.

The remainder of the paper is organized as follows: Section II introduces the VLP system model with RGB LEDs. Then, the CRLB derivations are performed in Section III. The numerical examples are presented in Section IV, which are followed by the concluding remarks in Section V.

\section{System Model}

Consider a VLP system that consists of LED transmitters at known locations (e.g., on the ceiling of a room) and VLC receivers at unknown locations. The VLC 
receivers estimate their locations by utilizing the signals emitted by the LED transmitters (i.e., self-positioning [11]). In this study, we consider a scenario in which each VLC receiver performs distance (range) estimation with each of the LED transmitters, and then determines its location based on those distance estimates [5]. Therefore, in this framework, accuracy of distance estimation is the main factor that determines the accuracy of location estimation [5, Sec. VI]. The purpose of this study is to determine the accuracy limits of distance estimation for VLP systems with RGB LEDs, which has not been investigated in the literature.

Consider an RGB LED transmitter at location $\boldsymbol{l}_{t} \in \mathbb{R}^{3}$ and a VLC receiver at location $\boldsymbol{l}_{r} \in \mathbb{R}^{3}$. The distance between the LED transmitter and the VLC receiver is represented by $x$, which is expressed as $x=\left\|\boldsymbol{l}_{r}-\boldsymbol{l}_{t}\right\|_{2}$. The RGB LED transmitters emits red, green, and blue signals simultaneously, which are denoted by $s_{i}(t)$ for $i \in\{r, g, b\}$. Then, the VLC receiver processes the incoming optical signals via three parallel photodetectors (PDs) corresponding to red, green, and blue signals (as shown in Fig. 1), and obtains the following electrical signals:

$$
y_{j}(t)=\sum_{i \in\{r, g, b\}} h_{j, i} s_{i}(t-\tau)+n_{j}(t)
$$

for $j \in\{r, g, b\}$ and $t \in\left[T_{1, j}, T_{2, j}\right]$, where $T_{1, j}$ and $T_{2, j}$ specify the observation interval for $\operatorname{PD} j, h_{j, i}$ is the channel attenuation factor between LED $i$ and PD $j$ $\left(h_{j, i}>0\right), \tau$ is the TOA parameter, and $n_{j}(t)$ is zeromean additive white Gaussian noise with a spectral density level of $\sigma_{j}^{2}$. The transmitted signals $s_{i}(t)$ are nonzero over an interval of $\left[0, T_{\mathrm{s}, i}\right]$ for $i \in\{r, g, b\}$, and they are assumed to be known by the VLC receiver. Also, the noise components, $n_{j}(t), j \in\{r, g, b\}$, are modeled as independent random processes as they are generated in different branches of the VLC receiver.

The TOA parameter in (1) can be expressed as

$$
\tau=\frac{x}{c}+\Delta
$$

where $c$ denotes the speed of light and $\Delta$ is the time offset between the clocks of the LED transmitter and the VLC receiver. For synchronous VLP systems, $\Delta=0$ whereas $\Delta$ is an unknown parameter for asynchronous VLP systems. As in [5], it is assumed that a coarse acquisition is performed such that the signal component in (1) resides completely in the observation interval $\left[T_{1, j}, T_{2, j}\right]$ for $j \in\{r, g, b\}$.

Considering a line-of-sight condition, the channel attenuation factors in (1) are given by [12]-[15]

$$
h_{j, i}=\frac{A_{j}(m+1)}{2 \pi x^{2}} \cos ^{m}(\phi) \cos (\theta) \Upsilon_{j, i}(\theta) g_{j}(\theta)
$$

for $i, j \in\{r, g, b\}$, where $A_{j}$ is the area of PD $j, m$ is the Lambertian order, $\phi$ is the irradiation angle, $\theta$ is the incidence angle, $\Upsilon_{j, i}(\theta)$ is the optical filter gain of PD $j$ for the signal coming from LED $i$, and $g_{j}(\theta)$ is the optical concentrator gain at PD $j$. By employing a suitable hemisphere lens as an optical concentrator, $\Upsilon_{j, i}(\theta) g_{j}(\theta)$ can be made almost independent of $\theta$, as discussed in [12], [13]; hence, it is defined as $\Upsilon_{j, i}(\theta) g_{j}(\theta) \triangleq \tilde{R}_{j, i}$ in the remainder of the manuscript.

As in [3]-[5], [16], it is assumed that the LED transmitter points downwards (which is a common scenario) and the VLC receiver points upwards such that $\phi=\theta$ and $\cos (\phi)=\cos (\theta)=\tilde{h} / x$, where $\tilde{h}$ denotes the height of the LED transmitter relative to the VLC receiver. In addition, it is assumed similarly to [3]-[5], [16] that $\tilde{h}$ is known by the VLC receiver; that is, possible locations of the VLC receiver are confined to a twodimensional plane (e.g., to the floor of a room). Although this assumption is made in order to facilitate theoretical analyses and provide intuitive and clear explanations for accuracy limits of distance estimation in VLP systems with RGB LEDs, it also holds in many practical scenarios; e.g., when the VLC receiver is attached to a warehouse vehicle or a robot (see Fig. 3 in [2]). Under these assumptions, the channel attenuation factors in (1) can be expressed as

$$
h_{j, i}=\frac{A_{j}(m+1)}{2 \pi x^{2}}\left(\frac{\tilde{h}}{x}\right)^{m+1} \tilde{R}_{j, i} \triangleq \gamma_{j, i} x^{-m-3}
$$

for $i, j \in\{r, g, b\}$, where $\gamma_{j, i}$ is defined as $\gamma_{j, i} \triangleq$ $A_{j}(m+1) \tilde{h}^{m+1} \tilde{R}_{j, i} /(2 \pi)$.

\section{THEORETICAL LIMITS}

In this section, accuracy limits of distance estimation are investigated for VLP systems with RGB LEDs under various scenarios.

\section{A. Scenario 1: Synchronous System with Known Chan- nel Attenuation Formula}

In this scenario, it is assumed that the LED transmitter and the VLC receiver are synchronized; hence, $\Delta=0$ in (2). In addition, the VLC receiver is assumed to know the channel attenuation formula in (4) with $\gamma_{j, i}$ 's being known constants. (In practice, $\gamma_{j, i}$ 's can be learned via calibration by placing the VLC receiver at known distances.) Under these assumptions, the log-likelihood function corresponding to the received signals in (1) can be expressed, based on (2) with $\Delta=0$ and (4), as follows:

$$
\begin{aligned}
\Lambda(x)=k- & \sum_{j \in\{r, g, b\}} \frac{1}{2 \sigma_{j}^{2}} \int_{T_{1, j}}^{T_{2, j}}\left(y_{j}(t)\right. \\
& \left.-\sum_{i \in\{r, g, b\}} \gamma_{j, i} x^{-m-3} s_{i}\left(t-\frac{x}{c}\right)\right)^{2} d t
\end{aligned}
$$

where $k$ is a constant that does not depend on $x$. 


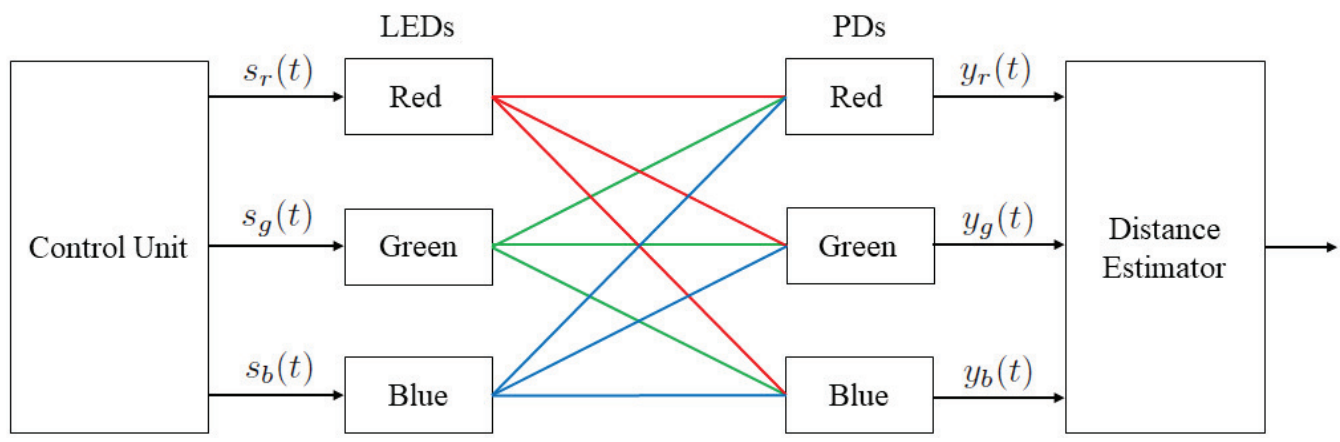

Fig. 1. VLP system model with RGB LEDs.

The CRLB provides a lower limit on MSEs of unbiased estimators and can be obtained from the loglikelihood function as follows:

$$
\mathrm{CRLB}_{1}=\left(\mathrm{E}\left\{\left(\frac{d \Lambda(x)}{d x}\right)^{2}\right\}\right)^{-1}
$$

From (5), the CRLB in (6) can be derived as

$$
\begin{aligned}
& \mathrm{CRLB}_{1}=\left(\sum _ { j \in \{ r , g , b \} } \frac { 1 } { \sigma _ { j } ^ { 2 } } \int _ { T _ { 1 , j } } ^ { T _ { 2 , j } } \left[x^{-m-4} \sum_{i \in\{r, g, b\}} \gamma_{j, i}\right.\right. \\
& \left.\left.\times\left((m+3) s_{i}(t-x / c)+(x / c) s_{i}^{\prime}(t-x / c)\right)\right]^{2} d t\right)^{-1}
\end{aligned}
$$

where $s_{i}^{\prime}(t)$ denotes the derivative of $s_{i}(t)$. After some manipulation, (7) can be expressed as

$$
\begin{aligned}
\mathrm{CRLB}_{1}= & \left((m+3)^{2} x^{-2 m-8} \kappa+\frac{x^{-2 m-6}}{c^{2}} \kappa^{\prime \prime}\right. \\
& \left.+\frac{2(m+3)}{c} x^{-2 m-7} \kappa^{\prime}\right)^{-1}
\end{aligned}
$$

where

$$
\begin{gathered}
\kappa \triangleq \sum_{j \in\{r, g, b\}} \frac{1}{\sigma_{j}^{2}} \sum_{i \in\{r, g, b\}} \sum_{l \in\{r, g, b\}} \gamma_{j, i} \gamma_{j, l} E_{i, l} \\
\kappa^{\prime} \triangleq \sum_{j \in\{r, g, b\}} \frac{1}{\sigma_{j}^{2}} \sum_{i \in\{r, g, b\}} \sum_{l \in\{r, g, b\}} \gamma_{j, i} \gamma_{j, l} E_{i, l}^{\prime} \\
\kappa^{\prime \prime} \triangleq \sum_{j \in\{r, g, b\}} \frac{1}{\sigma_{j}^{2}} \sum_{i \in\{r, g, b\}} \sum_{l \in\{r, g, b\}} \gamma_{j, i} \gamma_{j, l} E_{i, l}^{\prime \prime}
\end{gathered}
$$

with

$$
\begin{aligned}
& E_{i, l} \triangleq \int_{-\infty}^{\infty} s_{i}(t) s_{l}(t) d t \\
& E_{i, l}^{\prime} \triangleq \int_{-\infty}^{\infty} s_{i}(t) s_{l}^{\prime}(t) d t \\
& E_{i, l}^{\prime \prime} \triangleq \int_{-\infty}^{\infty} s_{i}^{\prime}(t) s_{l}^{\prime}(t) d t
\end{aligned}
$$

Various observations can be made based on (8)-(14). First, as expected, there is contribution to accuracy from all three colors. Second, distance related information contained in both the received signal strength (RSS) parameter and the TOA parameter is utilized in this scenario. To see this more clearly, suppose that the same intensity levels are transmitted from all the LEDs such that $s_{r}(t)=s_{g}(t)=s_{b}(t)$. Also, assume that $s_{i}(0)=s_{i}\left(T_{\mathrm{s}, i}\right)$ for $i \in\{r, g, b\}$, which is commonly the case for practical signals. Then, it is obtained from (10) and (13) that $\kappa^{\prime}=0$; hence, the CRLB in (8) becomes $\mathrm{CRLB}_{1}=\left((m+3)^{2} x^{-2 m-8} \kappa+x^{-2 m-6} c^{-2} \kappa^{\prime \prime}\right)^{-1}$. In this expression, the first term comes from the information obtained from the RSS parameter based on the known channel attenuation formula (as $\kappa$ is related to the total received power) and the second term is due to the TOA parameter (since $\kappa^{\prime \prime}$ is related to the time resolution; equivalently, the effective bandwidth of the signals). As a final observation, it can be shown that the CRLB formula in (8)-(14) covers the one in [5] as a special case if there exists only one LED at the transmitter and one PD at the receiver (cf. [5, Sec. III-A]).

\section{B. Scenario 2: Asynchronous System with Known Chan- nel Attenuation Formula}

In this scenario, it is assumed that the LED transmitter and the VLC receiver are asynchronous; namely, $\Delta$ in (2) is modeled as a deterministic unknown parameter. However, the VLC receiver is assumed to know the channel attenuation formula in (4) with $\gamma_{j, i}$ 's being known constants. In this case, the log-likelihood function corresponding to the received signals in (1) can be expressed via (4) as

$$
\begin{aligned}
\Lambda(x, \tau)=k- & \sum_{j \in\{r, g, b\}} \frac{1}{2 \sigma_{j}^{2}} \int_{T_{1, j}}^{T_{2, j}}\left(y_{j}(t)\right. \\
& \left.-\sum_{i \in\{r, g, b\}} \gamma_{j, i} x^{-m-3} s_{i}(t-\tau)\right)^{2} d t
\end{aligned}
$$


where $k$ is a constant that does not depend on $x$ or $\tau$. Then, the CRLB on distance estimation is given by ${ }^{1}$

$$
\mathrm{CRLB}_{2}=\left[\mathbf{J}_{\mathrm{F}}{ }^{-1}\right]_{11}
$$

where $\mathbf{J}_{\mathrm{F}}$ is the Fisher information matrix (FIM) defined as

$$
\mathbf{J}_{\mathrm{F}}=\left[\begin{array}{cc}
\mathrm{E}\left\{\left(\frac{\partial \Lambda(x, \tau)}{\partial x}\right)^{2}\right\} & \mathrm{E}\left\{\frac{\partial \Lambda(x, \tau)}{\partial x} \frac{\partial \Lambda(x, \tau)}{\partial \tau}\right\} \\
\mathrm{E}\left\{\frac{\partial \Lambda(x, \tau)}{\partial x} \frac{\partial \Lambda(x, \tau)}{\partial \tau}\right\} & \mathrm{E}\left\{\left(\frac{\partial \Lambda(x, \tau)}{\partial \tau}\right)^{2}\right\}
\end{array}\right] .
$$

From (15), the elements of the FIM in (17) can be calculated after some manipulation as

$$
\mathbf{J}_{\mathrm{F}}=\left[\begin{array}{cc}
(m+3)^{2} x^{-2 m-8} \kappa & (m+3) x^{-2 m-7} \kappa^{\prime} \\
(m+3) x^{-2 m-7} \kappa^{\prime} & x^{-2 m-6} \kappa^{\prime \prime}
\end{array}\right]
$$

where $\kappa, \kappa^{\prime}$, and $\kappa^{\prime \prime}$ are as defined in (9)-(11).

Based on (16) and (18), the CRLB on distance estimation can be calculated as

$$
\mathrm{CRLB}_{2}=\frac{\kappa^{\prime \prime} x^{2 m+8}}{(m+3)^{2}\left(\kappa \kappa^{\prime \prime}-\left(\kappa^{\prime}\right)^{2}\right)} .
$$

By comparing (8) and (19), it is noted that only the RSS parameter is utilized in this scenario since there is no synchronization between the transmitter and the receiver. In particular, if $\kappa^{\prime}=0$, then the CRLB in (19) becomes $\mathrm{CRLB}_{2}=\left((m+3)^{2} x^{-2 m-8} \kappa\right)^{-1}$, which corresponds to the first term in (8), as expected. Also, it is noted that the CRLB formula in (19) covers the one in $[5$, Sec. III-B] as a special case if there exists only one LED at the transmitter and one PD at the receiver.

\section{Scenario 3: Synchronous System with Unknown Channel Attenuation Formula}

In the final scenario, it is assumed that the LED transmitter and the VLC receiver are synchronized (i.e., $\Delta=0$ in (2)) but the VLC receiver does not know the channel attenuation formula in (4). Then, the loglikelihood function corresponding to the received signals in (1) can be expressed, based on (2) with $\Delta=0$, as follows:

$$
\begin{aligned}
\Lambda(\boldsymbol{\varphi})=k- & \sum_{j \in\{r, g, b\}} \frac{1}{2 \sigma_{j}^{2}} \int_{T_{1, j}}^{T_{2, j}}\left(y_{j}(t)\right. \\
& \left.-\sum_{i \in\{r, g, b\}} h_{j, i} s_{i}\left(t-\frac{x}{c}\right)\right)^{2} d t
\end{aligned}
$$

where $\varphi=\left[x h_{r, r} h_{r, g} h_{r, b} h_{g, r} h_{g, g} h_{g, b} h_{b, r} h_{b, g} h_{b, b}\right]^{T}$ is the vector of unknown parameters and $k$ is a constant that does not depend on $\varphi$.

In this scenario, the CRLB on distance estimation is stated as

$$
\mathrm{CRLB}_{3}=\left[\mathbf{J}_{\mathrm{F}}{ }^{-1}\right]_{11}
$$

${ }^{1}$ In (16) and (21), $[\mathbf{X}]_{11}$ denotes the element of matrix $\mathbf{X}$ at row 1 and column 1 . where $\mathbf{J}_{\mathrm{F}}$ is the FIM, which has a size of $10 \times 10$. The elements of $\mathbf{J}_{\mathrm{F}}$ are specified as follows:

$$
\mathbf{J}_{\mathrm{F}}=\left[\begin{array}{cc}
\mathrm{A} & \mathbf{B} \\
\mathbf{B}^{T} & \mathbf{C}
\end{array}\right]
$$

where $\mathrm{A}=\mathrm{E}\left\{\left(\frac{\partial \Lambda(\varphi)}{\partial x}\right)^{2}\right\}, \mathrm{B}$ is a $1 \times 9$ vector given by $\mathbf{B}=\left[\mathrm{E}\left\{\frac{\partial \Lambda(\varphi)}{\partial x} \frac{\partial \Lambda(\varphi)}{\partial h_{l, k}}\right\}\right]$ for $l, k \in\{r, g, b\}$, and $\mathbf{C}$ is a $9 \times 9$ matrix defined as $\mathbf{C}=\left[\mathrm{E}\left\{\frac{\partial \Lambda(\varphi)}{\partial h_{l, k}} \frac{\partial \Lambda(\varphi)}{\partial h_{n, m}}\right\}\right]$ for $l, k, n, m \in\{r, g, b\}$. Based on (20), the elements of $\mathbf{J}_{\mathrm{F}}$ in (22) can be specified as follows:

$$
\begin{aligned}
& \mathrm{A}=\frac{1}{c^{2}} \sum_{j \in\{r, g, b\}} \frac{1}{\sigma_{j}^{2}} \sum_{i \in\{r, g, b\}} \sum_{l \in\{r, g, b\}} h_{j, i} h_{j, l} E_{i, l}^{\prime \prime} \\
& \mathbf{B}=\left[\frac{1}{c \sigma_{l}^{2}} \sum_{i \in\{r, g, b\}} h_{l, i} E_{k, i}^{\prime}\right], \quad l, k \in\{r, g, b\} \\
& \mathbf{C}=\left[\frac{E_{k, m} \mathbb{1}_{\{l=n\}}}{\sigma_{l}^{2}}\right], \quad l, k, n, m \in\{r, g, b\}
\end{aligned}
$$

where $E_{k, m}, E_{k, i}^{\prime}$, and $E_{i, l}^{\prime \prime}$ are as defined in (12)-(14), and $\mathbb{1}_{\{l=n\}}$ is the indicator function, which is equal to one if $l=n$ and zero otherwise.

From (22)-(25), the CRLB in (21) can be obtained as

$$
\mathrm{CRLB}_{3}=\left(\mathrm{A}-\mathbf{B C}^{-1} \mathbf{B}^{T}\right)^{-1} .
$$

In this scenario, the distance related information in the TOA parameter is utilized since the system is synchronous but the channel attenuation formula is unknown. As a special case, if $E_{k, i}^{\prime}=0$ for all $k, i \in$ $\{r, g, b\}$, then $\mathbf{B}=\mathbf{0}$ and $\mathrm{CRLB}_{3}=1 / \mathrm{A}$. In this case, unknown channel attenuation factors, $h_{j, i}$ 's, do not affect the distance estimation accuracy. In all other cases, distance estimation accuracy is affected by the presence of unknown channel attenuation factors (as they influence how accurately the TOA information can be extracted)

\section{Numerical Results}

In this section, numerical examples are presented to investigate the CRLBs derived in Section III. A similar setting to that in [5] is considered; that is, the Lambertian order is set to $m=1$ and $\tilde{h}$ in (4) is taken as 2.5 meters. The areas of the PDs at the VLC receiver are set to $A_{j}=1 \mathrm{~cm}^{2}$ for $j \in\{r, g, b\}$, and the spectral density level of the noise components at different branches of the VLC receiver are $\sigma_{j}^{2}=1.336 \times 10^{-22} \mathrm{~W} / \mathrm{Hz}$ for $j \in\{r, g, b\}$ [3], [5]. The transmitted signals from the LEDs are modeled as [3]:

$$
s_{i}(t)=P_{o}\left(1-\cos \left(\frac{2 \pi t}{T_{s}}\right)\right)\left(1+\cos \left(2 \pi f_{i} t\right)\right)
$$

for $t \in\left[0, T_{s}\right]$ and $i \in\{r, g, b\}$, where $f_{i}$ is the center frequency specified as $f_{r}=0.9 f_{c}, f_{g}=f_{c}$, and $f_{b}=1.1 f_{c}$, and $P_{o}$ corresponds to the average emitted optical power 


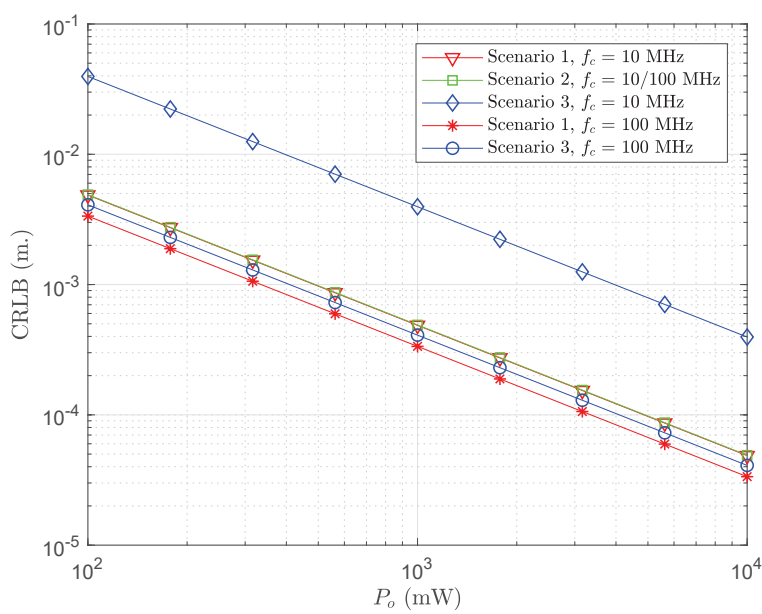

Fig. 2. CRLB versus $P_{o}$ for $x=5 \mathrm{~m}$. and $T_{s}=0.01 \mathrm{sec}$.

(i.e., source optical power). In addition, the $\tilde{R}_{j, i}$ terms in (4) are taken as $\left[\begin{array}{lll}\tilde{R}_{r, r} & \tilde{R}_{r, g} & \tilde{R}_{r, b}\end{array}\right]=0.4 \times\left[\begin{array}{lll}1 & 0.042 & 0.03\end{array}\right]$, $\left[\begin{array}{lll}\tilde{R}_{g, r} & \tilde{R}_{g, g} & \tilde{R}_{g, b}\end{array}\right]=0.4 \times\left[\begin{array}{lll}0.194 & 0.665 & 0.277\end{array}\right]$, and $\left[\begin{array}{llll}\tilde{R}_{b, r} & \tilde{R}_{b, g} & \tilde{R}_{b, b}\end{array}\right]=0.4 \times\left[\begin{array}{lll}0.009 & 0.084 & 0.421\end{array}\right]$, where $0.4 \mathrm{~mA} / \mathrm{mW}$ represents a coefficient related to the responsivity of the PDs as in [5], and the remaining numbers are adopted from eqn. (14) in [9].

First, the CRLBs (in meters) for the considered scenarios in Section III are plotted in Fig. 2 with respect to $P_{o}$ in (27) (equivalently, with respect to source optical power), where $x=5 \mathrm{~m}$. and $T_{s}=0.01 \mathrm{sec}$. It is noted that for small center frequencies (around $10 \mathrm{MHz}$ ), the CRLBs in Scenario 1 and Scenario 2 are almost the same since synchronization does not bring any additional benefits in this case. In other words, the distance related information contained in the RSS parameter is more significant than that in the TOA parameter. This can also be verified from the high CRLB values in Scenario 3 for $f_{c}=10 \mathrm{MHz}$ as only the TOA parameter is utilized in that scenario. As the center frequencies are increased, the TOA parameter becomes significant and the CRLB in Scenario 3 decreases rapidly. Since only the RSS information is used in Scenario 2, its CRLB does not depend on the center frequencies. On the other hand, the CRLB of Scenario 1 also decreases with increased center frequencies as it utilizes both the RSS and TOA parameters in distance estimation.

Fig. 3 illustrates the frequency dependencies of the CRLB expressions more explicitly, where $x=5 \mathrm{~m}$., $T_{s}=0.01$ sec., and $P_{o}=0.1$ are used. As the center frequencies of the transmitted signals are raised, the distance related information in the TOA parameter increases. Hence, the CRLB in Scenario 3, which only utilizes the TOA parameter, decreases with the center frequency parameter $f_{c}$ in Fig. 3. On the other hand, the CRLB in Scenario 2 does not change with the

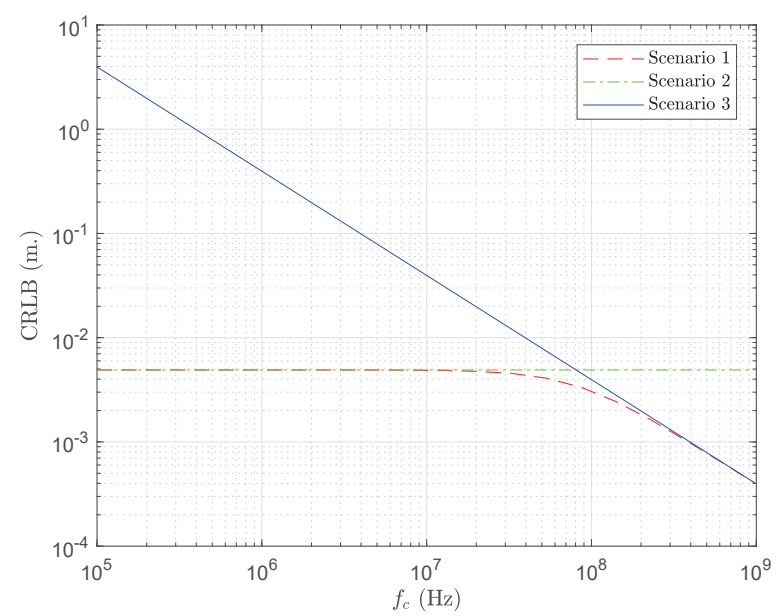

Fig. 3. CRLB versus $f_{c}$ for $x=5 \mathrm{~m} ., T_{s}=0.01 \mathrm{sec}$, and $P_{o}=0.1$.

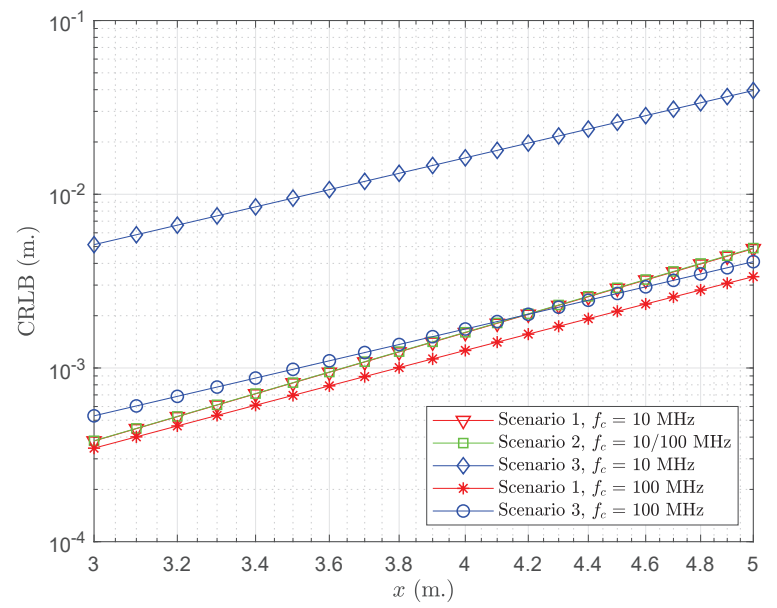

Fig. 4. CRLB versus $x$ for $P_{o}=0.1$ and $T_{s}=0.01 \mathrm{sec}$.

center frequencies, as noted before. Since both the TOA and RSS parameters are utilized in Scenario 1, the CRLB is almost constant for small $f_{c}$ 's (as the distance related information in the TOA parameter is insignificant compared to that in the RSS parameter in that region) and then starts decreasing with $f_{c}$ (as the distance related information in the TOA parameter gets significant).

Finally, the CRLBs are plotted versus the distance $x$ in Fig. 4, where $P_{o}=0.1$ and $T_{s}=0.01 \mathrm{sec}$. As expected, it is observed that the CRLBs increase with distance. However, the slopes of the CRLBs with respect to distance are not the same. The slope of the CRLB in Scenario 2 is higher than that in Scenario 3 since they are proportional to $x^{m+4}$ and $x^{m+3}$, respectively (considering the CRLBs in meters) based on the expressions in Sections III-B and III-C. On the other hand, the slope of the CRLB in Scenario 1 (see (8)) is almost the same 
as that in Scenario 2 for low center frequencies (as the RSS parameter is dominant in that case) and it is close to and higher than that in Scenario 3 for high center frequencies (as the TOA parameter is significant in that case, as well).

\section{CONCLUding RemarKs}

Performance limits of distance estimation have been investigated for VLP systems in the presence of RGB LEDs by considering different scenarios. In Scenario 1 and Scenario 2, a synchronous and an asynchronous system have been assumed, respectively, with a known channel attenuation formula at the VLC receiver. In Scenario 3, synchronism has been assumed but the channel attenuation formula has been modeled as unknown. Since both the TOA and RSS parameters are utilized in Scenario 1, it has the lowest CRLBs in all the cases. On the other hand, Scenario 2 achieves lower (higher) CRLBs than Scenario 3 for low (high) center frequencies (more generally, effective bandwidths).

\section{REFERENCES}

[1] M. F. Keskin, A. D. Sezer, and S. Gezici, "Localization via visible light systems," Proceedings of the IEEE, vol. 106, no. 6, pp. 1063-1088, June 2018.

[2] J. Armstrong, Y. Sekercioglu, and A. Neild, "Visible light positioning: A roadmap for international standardization," IEEE Communications Magazine, vol. 51, no. 12, pp. 68-73, Dec. 2013.

[3] T. Wang, Y. Sekercioglu, A. Neild, and J. Armstrong "Position accuracy of time-of-arrival based ranging using visible light with application in indoor localization systems," Journal of Lightwave Technology, vol. 31, no. 20, pp. 3302-3308, Oct. 2013.

[4] X. Zhang, J. Duan, Y. Fu, and A. Shi, "Theoretical accuracy analysis of indoor visible light communication positioning system based on received signal strength indicator," Journal of Lightwave Technology, vol. 32, no. 21, pp. 4180-4186, Nov. 2014.

[5] M. F. Keskin and S. Gezici, "Comparative theoretical analysis of distance estimation in visible light positioning systems," Journal of Lightwave Technology, vol. 34, no. 3, pp. 854-865, Feb. 2016.

[6] A. Sahin, Y. S. Eroglu, I. Guvenc, N. Pala, and M. Yuksel, "Accuracy of AOA-based and RSS-based 3D localization for visible light communications," in IEEE Vehic. Technol. Conf. (VTC), Boston, MA, Sep. 2015.

[7] E. Gonendik and S. Gezici, "Fundamental limits on RSS based range estimation in visible light positioning systems," IEEE Communications Letters, vol. 19, no. 12, pp. 2138-2141, Dec. 2015.

[8] M. F. Keskin, S. Gezici, and O. Arikan, "Direct and twostep positioning in visible light systems," IEEE Transactions on Communications, vol. 66, no. 1, pp. 239-254, Jan. 2018.

[9] E. Monteiro and S. Hranilovic, "Design and implementation of color-shift keying for visible light communications," Journal of Lightwave Technology, vol. 32, no. 10, pp. 2053-2060, May 2014.

[10] K. Choi, Y. Jang, M. Ju, and Y. Park, "Visible light communication with color and brightness control of RGB LEDs," ETRI Journal, vol. 35, no. 5, pp. 927-930, 2013.

[11] S. Gezici, "A survey on wireless position estimation," Wireless Personal Communications, vol. 44, no. 3, pp. 263-282, Feb. 2008.

[12] J. M. Kahn and J. R. Barry, "Wireless infrared communications," Proceedings of the IEEE, vol. 85, no. 2, pp. 265-298, Feb. 1997.

[13] W. Zhang, M. I. S. Chowdhury, and M. Kavehrad, "Asynchronous indoor positioning system based on visible light communications," Optical Engineering, vol. 53, no. 4, pp. 045 105-1045 105-9, 2014.
[14] P. Chvojka, S. Zvanovec, P. A. Haigh, and Z. Ghassemlooy, "Channel characteristics of visible light communications within dynamic indoor environment," Journal of Lightwave Technology, vol. 33, no. 9, pp. 1719-1725, May 2015.

[15] L. Li, P. Hu, C. Peng, G. Shen, and F. Zhao, "Epsilon: A visible light based positioning system," in 11th USENIX Symposium on Networked Systems Design and Implementation (NSDI), Seattle, WA, Apr. 2014, pp. 331-343.

[16] S.-H. Yang, E.-M. Jung, and S.-K. Han, "Indoor location estimation based on LED visible light communication using multiple optical receivers," IEEE Communications Letters, vol. 17, no. 9, pp. 1834-1837, Sep. 2013. 